S. Le Bras, "Business as usual ? Société de consommation et Grande guerre ", Histoire, économie et société, $n^{\circ} 3,2018$, p. 96-114

Ceci est une version intermédiaire du texte final. Elle contient probablement des coquilles

\title{
Business as usual ? Société de consommation et Grande Guerre. L'expérience des camions-bazars dans l'armée française
}

Stéphane Le Bras - Université Clermont-Auvergne

Adresse : Université Clermont-Auvergne, Boulevard Gergovia, 63000 Clermont-Ferrand

\begin{abstract}
Résumé :
Alors que les armées françaises s'installent dans la guerre de position, la question du ravitaillement prend des proportions inédites. Au cœur de cette problématique se trouve la thématique de la vie chère, de ses causes et des moyens d'y remédier. Pour ce faire, l'armée française met en place des structures commerciales innovantes, les camions-bazars. Censés répondre à une demande constante sur le front, ceux-ci ont surtout pour but de maintenir le moral des troupes et donc de participer à l'effort de guerre. Dans le même temps, ils témoignent d'une transformation du rapport des soldats à la consommation, diffusant de nouvelles pratiques et représentations. Dès lors, dans ce domaine également, la guerre s'apparente à un accélérateur de l'histoire.
\end{abstract}

\section{Summary:}

While the French armies settle down in the trenches warfare, the question of the provisioning takes new proportions. At the heart of this problem is the theme of the expensive life at the Front, its causes and the means to remedy it. In that order, the French army sets up innovative commercial structures, the camions-bazars. Supposed to respond to a constant demand on front, they aim as well at maintaining the morale of the troops and thus at participating in the war effort. At the same time, they prove a process of transformation of the soldiers' connection to the consumption, spreading new practices and representations. Thus, in this domain also, the war is similar to an accelerator of the history. 
S. Le Bras, "Business as usual ? Société de consommation et Grande guerre ", Histoire, économie et société, $n^{\circ} 3,2018$, p. 96-114

Dans leur Histoire anecdotique de la guerre parue en septembre 1915, Franc-Nohain et Paul Delay abordent l'importance des "services d'arrière " dans le ravitaillement des soldats français, un des facteurs primordiaux selon eux " des victoires ou des défaites des armées en campagne ${ }^{1}$ ». De manière circonstanciée, ils détaillent les circuits de ravitaillement règlementaires et réguliers (en vivres, en vêtements ou en munitions) des troupes stationnées dans la zone des armées. Toutefois, s'ils se montrent exhaustifs dans l'analyse d'un service déjà ancien ${ }^{2}$, ils oublient le ravitaillement parallèle qui se met en place au front dès la fixation de celui-ci à l'automne 1914. En effet, autour des cantonnements et des garnisons, le jeu commercial, articulé autour d'une offre et d'une demande de produits que les soldats ne peuvent se procurer par le biais des services de l'Intendance ${ }^{3}$, devient intense et prend une nouvelle dimension.

Cette situation est confirmée par les témoignages qui abondent depuis la troupe ou les autorités. Ainsi, le tome XI consacré à " La direction de l'arrière " de l'imposante somme Les Armées françaises dans la Grande Guerre expose la logistique du ravitaillement des soldats. Ses auteurs ${ }^{4}$ indiquent qu'outre la fourniture des denrées d'ordinaire (c'est-à-dire celles comprises dans la ration quotidienne et le paquetage du soldat), d'autres réseaux commerciaux coexistent afin de " fournir aux troupes les denrées et objets divers dont elles ont besoin ${ }^{5}$ ", marchandises que les soldats ne peuvent obtenir directement par les services de I'Intendance. Ces " achats importants", effectués dans "le commerce local " ${ }^{6}$, sont confirmés par les carnets ou les correspondances de poilus. L'aspirant Ferdinand Gillette raconte ainsi qu'au passage dans un village pendant une marche, le capitaine de sa compagnie fait arrêter ses hommes pour leur permettre " d'aller dans les épiceries et les bazars acheter ce qu'ils veulent ${ }^{7}$ ". De son côté, fin octobre 1914, Louis Lecomte, chef de pièce au $32^{\mathrm{e}}$ Régiment d'artillerie, explique qu'il profite d'une halte dans un petit bourg de l'Aisne pour se " réapprovisionner en tout ". Il détaille alors ses achats: charcuterie, pain, chocolat, vin, alcool, papier, cartes, tabac, sardines, crayon, carnet de note, almanach de la région, etc ${ }^{8}$.

Ces pratiques, attestées également dans la littérature de guerre ${ }^{9}$, témoignent de la persistance de préoccupations et de pratiques importées de l'arrière. II ne s'agit pas là d'une exception tant, dans d'autres domaines, ces logiques de transpositions de comportements collectifs et individuels depuis la sphère civile jusqu'au front sont avérées et bien connues ${ }^{10}$. Néanmoins, elles relèvent ici d'un champ d'analyse et d'interprétation original, celui de la consommation, de la diffusion de ses pratiques, de la permanence de ses logiques ou de ses

\footnotetext{
${ }^{1}$ Franc-Nohain et Paul Delay, Histoire anecdotique de la guerre, Fascicule 9 "Les Services d'arrière ", Paris, Lethielleux éd., 1915, p. 7.

${ }^{2}$ Voir à ce sujet la thèse de Francis Garcia, La carrière des intendants militaires de 1870 à 1914, soutenue à Bordeaux-Montaigne sous la direction de Jean-Paul Jourdan en mars 2015.

${ }^{3}$ Sur l'Intendance pendant la guerre, voir l'article précurseur de Pierre Barral dans Gérard Canini (dir.), Les Fronts invisibles, Nancy, PUN, 1984, p. 69-78.

${ }^{4}$ Il s'agit du général de division Ragueneau et des colonels d'infanterie Sisteron et Maugin.

${ }^{5}$ Ministère de la Guerre, Les Armées françaises dans la Grande Guerre, Tome XI, "La direction de I'arrière ", Paris, Imp. nationale, 1937, p. 140.

${ }^{6}$ Ibid., p. 249.

${ }^{7}$ Carnets de Ferdinand Gillette, Fonds privés familiaux, 20 juin 1916.

${ }^{8}$ Carnets de guerre de Louis Lecomte, Fonds privés familiaux, 27 octobre 1914.

${ }^{9}$ On retrouve des épisodes semblables chez Barbusse ou Dorgelès.

${ }^{10}$ Citons par exemple les travaux d'Alexandre Lafon sur les pratiques de sociabilité et de solidarité au front ( $L a$ Camaraderie au front (1914-1918), Paris, Armand Colin, 2014) ou ceux de Jean-François Jagielski et Thierry Hardier sur les loisirs aux armées (Oublier l'apocalypse ? Loisirs et distractions des Poilus pendant la Grande Guerre, Paris, Imago, 2014).
} 
S. Le Bras, "Business as usual ? Société de consommation et Grande guerre ", Histoire, économie et société, $n^{\circ} 3,2018$, p. 96-114

dérives. À cet égard, la Grande Guerre - loin de marquer une rupture ou une parenthèse s'inscrit au contraire dans la continuité d'un phénomène déjà appréhendé par François Caron pour la Belle Époque ${ }^{11}$, à savoir l'émergence de nouveaux modèles de consommation ${ }^{12}$. Mais ici, ceux-ci ne sont désormais plus seulement relatifs à la population parisienne ou aux élites de province. En effet, dans la nouvelle ère du "règne du consommateur ${ }^{13}$ ", le poilu semble s'apparenter à un client comme un autre, avec des besoins et des envies, deux notions fondamentales dans une société de consommation dont on souhaite ici interroger l'intrication dans une guerre qui, par certains aspects, semble en marquer une étape cruciale. Ce tournant est d'autant plus important que l'entrée en guerre correspond pour le commerce français à une profonde période de désorganisation (mobilisation, destruction, réquisitions, etc.) et à un défi notable en matière de réponse à de nouveaux enjeux, notamment patriotiques ${ }^{14}$.

Pour répondre à cette interrogation autour de l'articulation du conflit - aux impératifs en apparence fort éloignés de ceux des acteurs commerciaux (assurer l'écoulement d'une marchandise et faire des bénéfices) - avec la diffusion de nouvelles approches en matière de consommation, nous verrons pour quelles raisons et selon quelles modalités l'armée met en place des structures commerciales innovantes au front, pour ensuite appréhender les conséquences de ces pratiques dans l'optique de l'irruption, sur le front, d'une société dite " de consommation ".

\section{I. $\quad$ Approvisionnement et cherté de la vie. Deux préoccupations constantes}

Rédigé quelques années après l'armistice dans l'optique de présenter « les problèmes entièrement nouveaux " auxquels elles durent faire face pendant le conflit, le tome XI des Armées françaises dans la Grande Guerre insiste en particulier sur les nouvelles conditions d'un conflit que l'on croyait court dans un premier temps, mais qui poussa, par son extension, état-major et soldats à " progressivement s'adapter aux nécessités d'une guerre prolongée de stabilisation $\aleph^{15}$. II s'ensuit une redéfinition même de l'expérience combattante pour les autorités - par le cadre qu'elles organisent - et les soldats - par leur remarquable propension à s'y accommoder. Cette expérience du front, si elle est en partie construite par des moments de tensions et de brutalités que constituent les combats ${ }^{16}$, s'articule également autour d'une vie commune et collective durant laquelle les soldats ne sont pas confrontés directement à l'ennemi. En retrait des premières lignes, ils vivent alors une existence quotidienne qui sans être normale, prend des atours de normalité quand leurs préoccupations communes sont, entre autres, de participer aux travaux collectifs, entretenir le cantonnement, lire la presse, boire et manger, jouer aux cartes ou s'approvisionner auprès de marchands locaux. Or, à ce sujet, dès le tournant de 1915 se font ressentir "la raréfaction des marchandises " et sa conséquence inéluctable, "l'élévation des prix dans la zone des armées ${ }^{17}$. Cette hausse est en réalité la résultante de plusieurs facteurs.

\footnotetext{
${ }^{11}$ François Caron, "L'embellie parisienne à la Belle Époque : l'invention d'un modèle de consommation », Vingtième Siècle. Revue d'histoire, vol. 47, 1995, p. 42-57.

12 Pour une compréhension plus large de ces phénomènes à l'échelle nationale, voir les travaux d'Emmanuelle Cronier, que je remercie pour sa lecture patiente et attentive, ainsi que ses nombreux conseils. Voir par exemple "Les "recettes économiques de guerre" ou la bonne conscience ménagère ", in Caroline Poulain (dir.), Boire et Manger entre 1914 et 1918, Gand, Snoeck, 2016, p. 129-135.

${ }^{13}$ Charles Gide, Le Règne du consommateur, Paris, L. Larose et L. Tenin, 1910.

${ }^{14}$ Sur le sujet, voire François Bouloc, Les Profiteurs de guerre, Paris, Éd. Complexe, 2008, p. 113-159.

${ }^{15}$ Ministère de la Guerre, Les Armées françaises dans..., op. cit., p. X-XI.

${ }^{16}$ Voir Stéphane Audouin-Rouzeau et Annette Becker, 14-18, retrouver la guerre, Paris, Gallimard, 2000.

17 Ministère de la Guerre, Les Armées françaises dans..., op. cit., p. 249.
} 
S. Le Bras, "Business as usual ? Société de consommation et Grande guerre ", Histoire, économie et société, $n^{\circ} 3,2018$, p. 96-114

Tout d'abord, en période de guerre, surtout lorsqu'elle se prolonge, les marchés sont classiquement frappés par une pénurie générale, entraînant la raréfaction des denrées. Celleci est d'autant plus importante dans la zone des armées où les réseaux d'approvisionnement et les moyens de production sont particulièrement désorganisés par les combats, les mouvements de troupes et le départ d'une partie de la population. Ainsi, il n'est pas rare de voir dans les témoignages de soldats, des épisodes renvoyant à cette problématique. Si PierreRieul Diduan, caporal lot-et-garonnais, explique à sa famille que l'argent ne lui servira à rien au front puisque, déjà à la fin de l'été 1914 , selon ses informations, " on n'y trouve rien ${ }^{18}$ ", le caporal Maurice Sieklucki du $66^{\mathrm{e}} \mathrm{Rl}$ écrit de son côté que la Meuse est un " pays où ne nous trouvons rien " (17 avril 1916), regrettant quelques jours plus tard qu'il n'y ait " même pas une épicerie » (21 avril) ${ }^{19}$. Roland Dorgelès, pour sa part, regrette que, pour pouvoir s'approvisionner dans un commerce situé dans un village du front, il soit nécessaire de " faire la queue pour entrer [et] se battre pour être servi ", tandis les hommes s'empoignent ou supplient pour la dernière boite de conserve ${ }^{20}$. Cette situation s'explique par le fait que l'approvisionnement règlementaire, assuré par l'Intendance quotidiennement, soit considéré comme insuffisant, qualitativement voire quantitativement tout au long du conflit. Dès la fin de 1914, des plaintes s'élèvent à ce propos des rangs de l'armée : en novembre 1914, le Grand quartier général (GQG) fait savoir que les réserves en rations règlementaires ne sont pas suffisantes pour permettre aux commandants d'armée d'augmenter les cadences d'approvisionnement, comme le réclament leurs troupes ${ }^{21}$. Deux ans plus tard, les revendications sont plus explicites : dans le rapport d'une visite inopinée d'un général sur le front, il apparaît que " les hommes de la $2^{\mathrm{e}}$ compagnie du $33^{\mathrm{e}} \mathrm{RT}$ se plaignent de pas avoir suffisamment à manger ${ }^{22}$ ». En sous-texte, La Revue hebdomadaire dans un article consacré au ravitaillement reconnaît implicitement ces difficultés du ravitaillement : " Il faut voir de près un ravitaillement [...] pour se rendre compte des mille petites difficultés, des mille petits détails imprévus auxquels cette opération donne lieu ${ }^{23}$. "Difficultés qui régulièrement entraînent " réclamations, observations, refus, demandes ${ }^{24}$ " de la troupe envers leur officier d'approvisionnement, personnage central pris entre deux tensions, celle de la carence et de la revendication.

À ces soucis s'ajoutent ceux liés à l'acheminement des produits depuis les familles des poilus jusqu'au front. On le sait ${ }^{25}$, les colis postaux transitent en grande quantité vers la zone des armées depuis l'arrière, remplis de produits divers et variés comme l'indique cette carte postale écrite par un mère soucieuse à son fils, depuis Volvic en 1915: "Ta cousine t'a envoyé un paquet samedi, [...] il y a un peu de fromage, de chocolat et un petit flacon d'eau-de-vie. [...] Dis-moi si tu veux des molletières en étoffe, je t'en enverrai, je pourrai également $t^{\prime}$ envoyer une chemise plus chaude ainsi qu'une flanelle ${ }^{26}$. " Or, comme le font remarquer Franc-Nohain et $P$. Delay, dès les premiers mois de guerre, de nombreuses doléances et

\footnotetext{
${ }^{18}$ Correspondance de Pierre-Rieul Diduan, Fonds privés familiaux, 11 septembre 1914.

${ }^{19}$ Archives départementales d'Indre-et-Loire [ADIL désormais], 1 J 1086, Correspondance de Maurice Sieklucki, avril 1916.

${ }^{20}$ Roland Dorgelès, Les Croix de bois, Paris, 1919, p. 112-113.

${ }^{21}$ Service historique de la défense [SHD désormais], 16 N 2644, Grand quartier général (GQG), Direction de l'Arrière, Approvisionnement en vins et alcools, Lettre du Général commandant en chef, 4 novembre 1914.

${ }^{22}$ SHD, 19 N 38, Armées - Front occidental, I ère Armée, Rapports d'inspection sur le front, Note, mai 1916.

${ }^{23}$ "Le Ravitaillement », La Revue hebdomadaire, n³9, 23 septembre 1916, p. 535.

${ }^{24}$ Ibid., p. 540.

${ }^{25}$ Outre les publications d'A. Lafon ou J.-F. Jagielski et T. Hardier qui les évoquent, voir le récent ouvrage de Laurent Albaret, La Poste pendant la Première Guerre mondiale, Lyon, Éd. Yvert \& Tellier, 2016.

${ }^{26}$ Carte postale, Fonds privés de l'auteur, 27 janvier 1915.
} 
S. Le Bras, "Business as usual ? Société de consommation et Grande guerre ", Histoire, économie et société, $n^{\circ} 3,2018$, p. 96-114

récriminations relatives à ce service postal émergent, tant en ce qui concerne le retard des acheminements, que les pertes ou les dégradations ${ }^{27}$. Cette situation est confirmée par plusieurs témoignages, tel P.-R. Diduan déçu de la réception d'un colis dont les victuailles sont gâtées ou d'une médaille dont on lui annonce l'expédition, mais qu'il ne reçoit pas ${ }^{28}$. D'ailleurs, dans un reportage sur le service de la Poste aux armées, si la revue Lectures pour tous se veut rassurante pour les familles de poilus, insistant sur l'exigence de résultats d'un tel service et sur ses succès, elle reconnaît également des difficultés dans l'organisation face à l'afflux de correspondance ${ }^{29}$. De fait, les mécontentements et agacements liés au service postal, tout comme la satisfaction de recevoir un paquet - quel que soit son contenu - mettent en exergue la permanence d'une préoccupation représentée par l'acquisition de nouveaux produits et objets, sources de confort matériel et moral. C'est en substance ce que décrit $L a$ Revue hebdomadaire :

Une large part du bien-être matériel de la troupe est confiée [à l'Intendant en charge du ravitaillement]. Par son ingéniosité, il peut apporter aux hommes de sa formation bien des petites satisfactions. [...] Jamais on ne donnera trop ni assez bien au magnifique combattant de la guerre ; mais il est permis de constater que le service responsable [de l'approvisionnement] s'est montré à la hauteur de sa lourde tâche. ${ }^{30}$

En dépit de ses aléas, ce double réseau d'approvisionnement et de fourniture est d'autant plus important et se doit d'être le plus efficace possible que le front est très rapidement colonisé par une foule de commerçants exploitant les soldats, les mercantis.

À cet égard, dès le début 1915 , le ministre de la Guerre, à l'aune des rapports qui lui sont transmis, s'émeut que, « dans les localités situées en arrière du front, les marchands de comestibles et les débitant vendent leurs produits aux troupes à des prix qui paraissent véritablement excessifs ${ }^{31}$. " L'été suivant, il réitère ses inquiétudes, soulignant que dans la zone des armées, " le commerce local impose à la consommation des troupes des conditions de prix, dont l'élévation excessive semble doublement regrettable ${ }^{32}$ ». Quelques mois plus tard, Édouard Herriot, sénateur-maire de Lyon, propose, de développer la production dans sa localité, afin « de contribuer, par un apport notable de denrées, à l'abaissement des prix des vivres et objets d'usage courant mis en vente dans les cantonnements " avec comme objectif d'" atténuer l'écart entre l'offre et la demande " comme le préconise un rapport rédigé par un lieutenant-colonel à la suite d'une enquête sur la question des prix dans la zone des armées $^{33}$. Au même moment, lors d'une discussion sur le relèvement de la solde au Sénat, l'élu de Haute-Saône et rapporteur de la commission de l'armée Jules Jeanneney évoque le risque que " la solde nouvelle des troupes devienne, pour des commerçants sans scrupule, le moyen d'accroitre des bénéfices injustifiés". " propose alors d'émettre un permis de stationnement et de circulation pour les « mercantis " qu'un autre sénateur, Adrien Gaudin

\footnotetext{
${ }^{27}$ Franc-Nohain et Paul Delay, Histoire anecdotique...op. cit., p. 106 et suiv.

${ }^{28}$ Correspondance de P.-R. Diduan, Fonds privés familiaux, 6 juin et 9 mai 1915.

29 "La Poste aux armées », Lectures pour tous, 15 décembre 1915, 447-456

30 " Le Ravitaillement ", La Revue hebdomadaire, art. cit., p. 535.

${ }^{31}$ SHD, 16 N 2644, arch. cit., Lettre du ministre de la Guerre à la Direction de l'Arrière, 17 février 1915.

32 Ibid., 19 août 1915.

${ }^{33}$ SHD, 16 N 146, GQG, $1^{\text {er }}$ Bureau, Organisation renforts, Lettre du GQG au ministre de la Guerre, 14 novembre 1915.
} 
S. Le Bras, "Business as usual ? Société de consommation et Grande guerre ", Histoire, économie et société, $n^{\circ} 3,2018$, p. 96-114

de Villaine, associe à des "voleurs et rastaquouères des marchés et spéculations de la guerre ${ }^{34}$. »

Cette figure du mercanti est relativement bien connue, tant en partie grâce aux travaux universitaires ${ }^{35}$ que par les publications et témoignages de l'époque. Le Dictionnaire des termes militaires et de l'argot poilu paru en 1916 le campe sobrement comme un " marchand à la suite des armées, sur les lignes d'arrière ${ }^{36} "$, tandis que l'hebdomadaire satirique La Baïonnette lui consacre un numéro spécial en octobre de la même année ${ }^{37}$. Les mercantis dans toute leur diversité - y sont représentés comme des personnages cupides, cyniques et veules, coupables de toutes les compromissions pour "tondre " le poilu et le déposséder d'une partie de son pécule. Ce sont souvent des néo-commerçants, civils ayant décidé de se tourner vers un nouvel emploi, comme le dépeint Dorgelès dans ce village du front où « tout le monde est marchand ", où " chaque maison est une boutique " ${ }^{38}$. Si le constat est abrupt, il est en partie confirmé par un rapport de la Seconde division britannique qui estime qu'une maison sur cinq dans le bourg de Caestre (Nord), est un estaminet ${ }^{39}$. Les mercantis recouvrent aussi l'ensemble des commerçants patentés qui se sont reconvertis dans l'approvisionnement des millions de soldats français ou étrangers qui affluent vers les zones de combat. Si l'on suit Dorgelès encore une fois, on retrouve dans ce village où cantonne son unité, un charcutier vendeur de peignes, une institutrice débitrice de saucisses et un épicier spécialisé dans les couronnes mortuaires ${ }^{40}$. On le voit, ici comme dans l'ensemble des reportages ou récits sur le sujet, les mercantis, ces " exploiteurs ${ }^{41}$ ", proposent toutes sortes de marchandises depuis les victuailles jusqu'aux vêtements, en passant bien sûr par l'inépuisable pinard, souvent trafiqué comme s'en moque une carte postale des années 1917-1918 (doc 1), mais toujours d'une meilleure qualité que celui fourni par l'Intendance ${ }^{42}$.

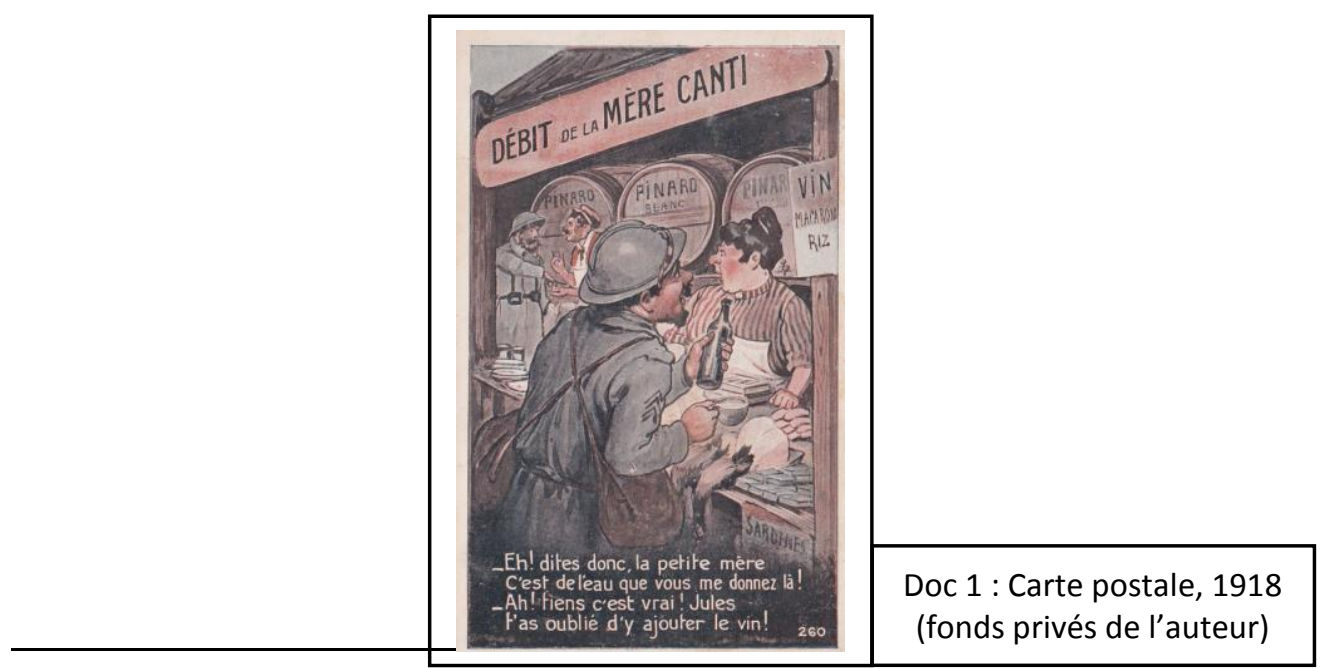

${ }^{34}$ Journal officiel de la République française [JORF désormais], Débats parlementaires, Sénat, 14 octobre 1915, p. 557 et 565 .

${ }^{35}$ F. Bouloc, Les Profiteurs de guerre..., op. cit. ; Jean-Louis Robert, "The image of the profiteer ", in J.-L. Robert et Jay Winter (dir.), Capital cities at war, London, Paris, Berlin, 1914-1919, Cambridge, Cambridge Univ. Press, 1997, p. 104-132.

${ }^{36}$ Dictionnaire des termes militaires et de l'argot poilu, Paris, Larousse, 1916, p. 191.

37 "Les Mercantis », La Baïonnette, n69, 26 octobre 1916.

${ }^{38}$ R. Dorgelès, Les Croix de bois, op. cit., p. 110.

${ }^{39}$ Craig Gibson, Behind the Front. British soldiers and French civilians, 1914-1918, Cambridge, 2014, p. 291.

${ }^{40}$ R. Dorgelès, Les Croix de bois, op. cit, p. 109-110.

${ }^{41}$ "Les convois auxiliaires d'ambulances automobiles ", La Revue hebdomadaire, 4 mars 1916, p. 120

42 SHD, 16 N 2644, arch. cit., Lettre de la Direction de l'Arrière au sous-secrétaire d'État du Ravitaillement et de I'Intendance, 4 sept. 1915. C'est le cas également pour un autre produit particulièrement prisé, le pain. Cf. SHD, 18 N 32, Groupes d'armées, Nord, Intendance et étapes, Vivres, coopératives, divers, Dossier « Ravitaillement ", Note du GQG, mars 1916. 
S. Le Bras, "Business as usual ? Société de consommation et Grande guerre ", Histoire, économie et société, $n^{\circ} 3,2018$, p. 96-114

Parfois, les soldats eux-mêmes participent au phénomène et s'improvisent revendeur si l'on en croit P.-R. Diduan, regrettant l'attitude de certains de ses camarades: "Beaucoup reçoivent des journaux et au repos on peut en acheter, ils sont vendus $0,10 \mathrm{~F}$ par des artilleurs qui les achètent 0,05 , tu vois que même à la guerre certains font du commerce ${ }^{43}$ »

Véritable marché, la zone des armées s'apparente à " une zone de vente " où " chacun [veut] prélever, au détriment du soldat, des bénéfices exagérés ${ }^{44}$. C'est un constat doublement confirmé : à la fois par le caporal Sieklucki prêt à acheter " même à prix d'or »"5 tout ce qu'il peut trouver, signe du renchérissement continu de la vie à proximité du front, que par diverses enquêtes militaires dont une menée par exemple sur « le prix de vente du fromage et particulièrement du fromage de Camembert » en octobre $1915^{46}$. C'est dans ce contexte qu'en août 1915, le député républicain modéré Emmanuel Brousse interpelle le ministre de la Guerre en lui demandant « quelles mesures il compte prendre pour que dans la zone des armées nos soldats ne soient plus exploités par des mercantis vendant tout à des prix exagérés ${ }^{47}$. " II reprend là un questionnement brûlant, qui revient régulièrement au Parlement ${ }^{48}$. Les autorités militaires, elles, s'alarment d'une situation qui « ne pouvait laisser le commandement indifférent ${ }^{49} "$.

Dès lors, des mesures s'imposent car, incontestablement, à partir du tournant de 1915, la question de la cherté de la vie au front devient un enjeu et une préoccupation majeurs pour l'ensemble des acteurs du conflit. Si les premières décisions visent à mettre en place, avec l'aide des préfets, la «taxation de certaines denrées [...] en consignant à la troupe les commerçants qui dépassaient les tarifs fixés ${ }^{50} "$, c'est surtout une organisation nouvelle - et innovante - de réapprovisionnement des soldats qui voit le jour fin janvier 1915 : les camionsbazars, dont l'instauration est justifiée dans Les Armées françaises dans la Grande Guerre par « la lutte contre les mercantis ${ }^{51}$ ».

\section{Les camions-bazars : innovation et modernité commerciales au front}

En réalité, dans un premier temps, dès la mi-janvier 1915, ce sont des wagons-bazars qui sont organisés par le commandement. Ceux-ci sont, en nombre limité (deux), « joints chaque jour à chacun des trains de ravitaillement quotidien " qui alimentent les armées cantonnées au front ${ }^{52}$. Une première circulaire fixe les conditions d'opération de ces nouveaux moyens d'approvisionnement :

\footnotetext{
${ }^{43}$ Correspondance de P.-R. Diduan, Fonds privés familiaux, 3 mai 1915.

${ }^{44}$ Ministère de la Guerre, Les Armées françaises dans..., op. cit., p. 249.

${ }^{45}$ ADIL, $1 \mathrm{~J}$ 1086, Correspondance de Maurice Sieklucki, 21 avril 1916.

${ }^{46}$ SHD, 16 N 146, arch. cit., " Dossier P.P. ", Lettre de la Direction de l'Arrière, 15 octobre 1915.

47 JORF désormais, Débats parlementaires, Chambre des députés, question écrite $n^{\circ} 4271,26$ août 1915, p. 1410.

${ }^{48}$ Voir par exemple la « proposition de résolution tendant à organiser le commerce, à règlementer la vente et à établir la taxation des marchandises de première nécessité vendues aux troupes sur le front et dans la zone des armées ", JORF, Chambre des députés, Impressions, n¹829, 22 février 1916.

${ }^{49}$ Ministère de la Guerre, Les Armées françaises dans..., op. cit., p. 249.

${ }^{50} I d$. Mais ses effets sont très limités, en raison de la complexité du mécanisme comme l'explique Charles Gide dans " La guerre et l'organisation nationale de l'alimentation », Revue d'économie politique, janv.-fév. 1916, p. $1-17$

${ }^{51}$ C'est le titre même d'un chapitre (numéro 10) de la section consacrée au ravitaillement en vivres, p. 249.

52 SHD, 18 N 32, arch. cit., Dossier "Taxation des denrées, coopératives, wagons-bazars \& camions-bazars, fraudes ", Note du GQG aux généraux commandants d'armée, généraux directeurs des étapes et services, commissaires régulateurs, 13 janvier 1915.
} 
S. Le Bras, "Business as usual ? Société de consommation et Grande guerre ", Histoire, économie et société, $n^{\circ} 3,2018$, p. 96-114

Aménagés par les soins de négociants, choisis par le Directeur des étapes et des services, [ils] contiendront de menus objets de mercerie, bonneterie, épicerie, papeterie, confiserie, articles de Paris, qui seront vendus aux soldats pendant l'arrêt du train à la gare de ravitaillement ${ }^{53}$.

En assumant le choix des partenaires commerciaux et des produits avalisés par le commandement, l'armée cherche à garder la main sur une pratique nouvelle, à savoir l'intrusion, légitimée par les autorités militaires, de sociétés à fondements capitalistes au cœur même de l'expérience combattante. Cela explique les nombreuses contraintes qui portent sur les candidats, afin d'éviter les dérives constatées jusque-là chez les commerçants du front. Ainsi, outre le choix du négociant, le Directeur des étapes et services (DES) devra s'entendre avec ce dernier pour "fixer la nature des objets à vendre " et "les limites de prix ", sans oublier de penser à l'organisation matérielle du service, afin d'éviter « que le système proposé ne dégénère en une sorte de marché ouvert à tout venant ". L'objectif est dès lors clairement édicté en fin de circulaire : "permettre [la] satisfaction rapide des besoins " de la troupe, notamment en produits permettant d'améliorer le quotidien des soldats. Enfin, afin d'éviter tout dysfonctionnement ou toute tentative de la part d'intermédiaires marrons de profiter de la situation, le nom des commerçants patentés par l'armée, ainsi que leurs représentants chargés de vendre les produits, seront notifiés dans l'ensemble des gares régulatrices et de ravitaillement du réseau en question ${ }^{54}$.

Très rapidement, en raison de l'encombrement du réseau ferré et parce qu'on envisage de l'étendre à l'ensemble des forces armées, le système est modifié. Fin janvier 1915, les commerçants appointés par l'armée pour approvisionner les troupes sont autorisés " à établir un entrepôt dans la localité où cantonne le parc de réserve automobile de l'armée ${ }^{55}$. » Depuis cet entrepôt, le gérant assurera l'envoi des commandes vers sa maison-mère et gèrera l'approvisionnement des troupes par le biais, désormais, d'automobiles. Celles-ci " partiront tous les jours suivant un itinéraire fixé par le DES " afin de desservir les cantonnements. Chaque automobile, aménagée pour la vente, sera conduite par un militaire, auquel sera associé un représentant du commerçant ${ }^{56}$, chargé $\mathrm{d}^{\prime}$ assurer avec "honorabilité » et " discrétion » un service pour lequel on fixe également le temps maximum passé dans chaque cantonnement.

Ce nouveau système de commercialisation, on le voit, participe d'un triple impératif. Tout d'abord, celui d'offrir un service au soldat, afin de répondre à un besoin, à savoir la satisfaction d'un ravitaillement efficace et bon marché. À cet effet, on prend donc une mesure de rationalisation des transactions commerciales au front, transactions assurées par un spécialiste de ces activités (le négociant) et légitimés par les autorités militaires (le DES). Par ailleurs, par le biais de ces dispositions, il s'agit d'encadrer fermement une pratique qui peut, comme en témoignent les dérives auxquelles se livrent les mercantis, devenir rapidement nuisible. C'est pour cela que de nombreuses clauses injonctives sont incorporées. Outre I'honorabilité et la discrétion, d'autres mesures coercitives (interdiction de léguer ou céder à un tiers tout ou partie du contrat pour le négociant ; possibilité de résiliation sur simple avis ; fixation des trajets ; validation des articles et de leurs prix) sont ainsi incluses dans les contrats,

\section{${ }^{53} / d$.}

${ }^{54}$ Ibid., Note aux commissions de réseau Nord, Est, PLM, État, 13 janvier 1915.

$55 \mathrm{lbid}$., Note du GQG aux généraux commandants d'armée, généraux directeurs des étapes et services, commissaires régulateurs, 23 janvier 1915.

${ }^{56}$ Début février, une note du GQG propose néanmoins un aménagement dans le cas où aucun civil ne pourrait être recruté. Des soldats pourraient alors être mis à disposition des négociants (note du GQG, 5 février 1915). Fin 1915, un contrat-type indique que le nombre de ces vendeurs s'élève à trois. 
S. Le Bras, "Business as usual ? Société de consommation et Grande guerre ", Histoire, économie et société, $n^{\circ} 3,2018$, p. 96-114

sans compter la nécessaire prudence en matière de diffusion de renseignements militaires. Ainsi, dans une circulaire mi-février, il est clairement notifié qu'aucun état ou note fournissant des informations de localisation ne pourra être établi, tandis que la liste des cantonnements et l'emplacement des troupes ne seront jamais communiqués à l'avance, les trajets étant fixés du soir pour le lendemain ${ }^{57}$. Surtout, ce nouveau réseau fait montre de la grande capacité d'adaptation de l'armée. À la fois dans la conception même du système que, comme le démontrent les circulaires entre mi-janvier et mi-février, dans son ajustement, par petites touches, aux conditions exceptionnelles du front, l'émergence de ce nouveau service permet de mesurer le souci des autorités d'articuler les besoins des soldats, l'efficacité des prestations et le contexte de guerre.

Dans la pratique, des conventions sont donc passées entre une armée, représentée par son général DES, et les sociétés distributrices. Fin 1915, le GQG diffuse à l'ensemble des généraux commandants d'armées et des généraux DES une convention-type ${ }^{58}$. On y apprend que le service est divisé en deux catégories (" épicerie " et "bazar ") et que les produits doivent être de "bonne qualité ", qualité que l'autorité militaire se réserve le droit d'analyser à tout moment. Les entrepôts sont mis gracieusement à disposition des sociétés commerciales qui les aménagera pour entreposer les marchandises. Cet aménagement pourra se faire avec l'aide de l'armée, mais sera, lui, remboursable (main-d'œuvre et matériaux). Les marchandises, dont les commerçants seront responsables, seront expédiées à leurs frais, jusqu'à la gare la plus proche ; l'armée s'engageant à favoriser « dans la plus large mesure du possible la bonne exécution et la rapidité des transports. " Les transports vers les soldats s'effectueront par le biais d'automobiles, dont le nombre minimum par entrepôts sera de trois, chiffre pouvant augmenter en vertu des besoins. Le trajet est pour sa part strictement fixé d'un jour sur l'autre, avec heure d'arrivée et de départ dans les cantonnements. De son côté, le personnel sera recruté - hormis les conducteurs - chez les civils dont le choix est rigoureusement encadré afin d'éviter les abus et les dérives (certificat de bonnes mœurs, carte d'identité avec photo, en règle avec la loi militaire, nationalité française). Dans le cas où ce recrutement ne serait pas possible, des soldats du Service auxiliaire ou de réserve de la Territoriale, ayant les compétences professionnelles pour s'y conformer, pourront être détachés afin de servir à l'entrepôt (gérant, caissier, manutentionnaire) ou dans les automobiles (vendeurs). Les tarifs enfin seront validés par le DES, tous les 15 jours, et largement diffusés dans les cantonnements par le biais de catalogues ou de prix-courants ( 5.000 pour la maison « Damoy » fin $\left.1915^{59}\right)$. Fort logiquement, certains interdits sont imposés (transport de lettres ou de paquets, divulgation des mouvements ou de la localisation de troupes, vente à crédit) et l'armée se réserve le droit de retirer camions et soldats, de suspendre la vente ou de rompre, sans justification, le contrat. Dans certains cas, les soldats pourront s'approvisionner directement auprès des camions-bazars, dans d'autres, un officier d'approvisionnement sera chargé de se rendre au lieu de passage pour acheter les objets désirés par les soldats de son unité.

\footnotetext{
${ }^{57}$ Ibid., Note du GQG aux généraux commandants d'armée, généraux directeurs des étapes et services, 12 février 1915.

58 Ibid., Note du GQG, 10 décembre 1915.

${ }^{59}$ SHD, 19 N 964, Armées - Front occidental, Ve Armée, Direction des étapes et services, Questions diverses traitées par l'Intendance, Dossier «Camions-bazars » $n^{\circ} 3$, Lettre de « J. Damoy » à la direction de l'Intendance de la Ve Armée, 30 octobre 1915.
} 
S. Le Bras, "Business as usual ? Société de consommation et Grande guerre ", Histoire, économie et société, $n^{\circ} 3,2018$, p. 96-114

En dépit de ces nombreuses contraintes ${ }^{60}$, la collaboration entre partenaires commerciaux et militaires semble être profitable à tout le monde, d'où son attractivité. Ainsi, dès janvier 1915, "La Ruche picarde " société anonyme amiénoise alors en pleine expansion ${ }^{61}$, propose ses services à la $\|^{\mathrm{e}}$ Armée, alors stationnée dans la région. Elle dispose d'un atout conséquent avec la présence dans la région de 25 succursales de vente encore en activité après l'entrée en guerre et surtout d'un entrepôt central à Amiens ${ }^{62}$. Autre avantage pour les autorités militaires, elle s'engage à ce que " les prix de vente [soient] ceux en usage dans les maisons de vente de la société, sans aucune majoration ", positionnement encore possible à cette époque car l'ensemble de l'acheminement des denrées est assumé financièrement par l'armée (ce qui ne sera plus le cas à la fin de l'année) ${ }^{63}$. Par une note du QG de la II Armée, "La Ruche picarde " est autorisée début février à assurer un service de camions-bazars à l'ouest d'une ligne d'une centaine de km allant de La Cauchie (Pas-de-Calais, au sud d'Arras) à Machemont (Oise, au nord de Compiègne) ${ }^{64}$ afin d'approvisionner les cantonnements qui s'y trouvent. Plus à l'est, c'est la société parisienne « Julien Damoy » qui assure l'approvisionnement de la V' Armée par camions-bazars depuis décembre 1915. Les prix courants distribués sur le front permettent de mesurer la diversité des produits mis à disposition des soldats ${ }^{65}$ : produits de consommation courante (café ; confiture ; pâtes ; conserves de légumes, de viandes ou de poissons ; vins au litre) y côtoient des denrées plus rares et moins ordinaires (pâtisseries, fruits au sirop ; chocolat ; vins fins) et des produits de « bazar » (parfumerie ; jeux ; papeterie ; mercerie ; ceintures ; bretelles ; etc.). Les archives de ce partenariat commercial nous indiquent également le soin apporté à l'emplacement de l'entrepôt (à proximité d'une voie ferrée et d'une route), l'aménagement de l'intérieur du camion-bazar, le nombre d'employés du service ainsi que les itinéraires quotidiens des automobiles. Ainsi, au printemps 1916, depuis l'entrepôt sis à proximité de la gare des Ventaux (Marne, au nord de Savigny ; choisi à l'automne 1915 après différentes études) ${ }^{66}$, à une dizaine de kilomètres des premières lignes, rayonnent quatre autos-bazars qui effectuent un circuit quotidien, couvrant sur la semaine près de $80 \mathrm{~km}$ le long du front et visitant deux fois par semaines les « centres de vente " où se rendent des délégués de soldats avec une liste précise des objets à acquérir ${ }^{67}$.

L'échange régulier de correspondance entre la grande maison parisienne et le DES ou I'Intendance permet de mettre en relief le perfectionnement régulier d'un service qui s'illustre par sa modernité dans un cadre original, celui d'une guerre de position. Ainsi, à l'origine, en juillet 1915, les prestations de "J. Damoy " sont assurées de manière expérimentales par le biais des wagons-bazars et uniquement limitées au $18^{\mathrm{e}}$ Corps de la Ve Armée. On apprend alors que le système est semblable à celui des ventes par correspondance telle que pratiquées par les grandes maisons de commerce parisiennes (Bon marché, Félix Potin, Samaritaine, etc.)

\footnotetext{
${ }^{60}$ Auxquelles il faut ajouter les difficultés pour de nombreuses sociétés à fonctionner comme en témoigne l'enquête menée pour la fourniture de la Ve Armée fin janvier 1915. Cf. SHD, 19 N 964, arch. cit., Lettre de l'adjoint à l'Intendance à l'Intendant général, $1^{\text {er }}$ février 1915.

${ }^{61}$ Pierre Caloin, De l'abeille au mammouth. La Ruche picarde, un siècle d'histoire (1895-1995), Amiens, Martelle Ed., 1995.

62 SHD, 19 N 294, Armées - Front occidental, ॥e Armée, Premier Bureau, Dossier " Instances », Liste des succursales en exercice de la Ruche picarde, s.d.

${ }^{63}$ Ibid., Lettre de l'Intendant militaire de la II Armée au DES, 29 janvier 1915.

${ }^{64}$ Ibid., Note du QG de la II Armée, février 1916.

${ }^{65}$ SHD, 19 N 964, arch. cit., Dossier « Camions-bazars » n4, Prix courants, $1^{\mathrm{er}}$ au 15 décembre 1915.

66 Dans la seconde moitié de 1916, cet entrepôt est déménagé à Fismes, où la Ve Armée installe son parc automobile, puis en juin 1917, au gré du déplacement des armées, à Bouleuse, au sud de Reims.

${ }^{67}$ SHD, 19 N 964, arch. cit., Dossier « Camions-bazars » $n^{\circ} 4$, Horaires et carte des circuits des autos-bazars de la maison « J. Damoy », note « Auto-Bazars » (s.d.), correspondance, 1915-1916.
} 
S. Le Bras, "Business as usual ? Société de consommation et Grande guerre ", Histoire, économie et société, $n^{\circ} 3,2018$, p. 96-114

depuis la seconde moitié du XIX ${ }^{e}$ siècle : une commande par unité est établie, relayée par l'officier d'approvisionnement sur un bon de commande ${ }^{68}$. Une semaine plus tard environ, un wagon-bazar transporte les produits jusqu'à la gare de ravitaillement destinataire ${ }^{69}$. C'est là un système qui n'est pas sans causer de problèmes : retards dans la transmission des ordres, encombrement des voies, erreurs de commande, obligation de venir chercher les produits commandés, diffraction des paiements, etc. C'est ce qui explique le passage, à la grande satisfaction de "J. Damoy", aux camions-bazars, système d'exploitation beaucoup plus moderne, souple et commode. C'est dans cette optique également qu'un soin tout particulier est apporté aux choix des soldats affectés au service: une vingtaine environ pour le commerce, entre 10 et 15 pour le transport selon les périodes. Ici encore, " J. Damoy » et les autorités s'entendent sur la nécessité d'avoir des hommes capables et expérimentés, dans les pratiques commerciales cette-fois-ci. Signe de ce souci, sur les demandes pressantes de «J. Damoy ", I'Intendance militaire affecte à la gestion de l'entrepôt des Ventaux, deux sousofficiers, employés de "J. Damoy" dans le civil70. Par ailleurs, le DES, en amont de la constitution du service aux Ventaux, active ses réseaux dès l'été 1915 pour se procurer une main-d'œuvre compétente: comptable, chef-magasinier, professions des industries de l'alimentation, emballeur ${ }^{71}$.

L'objectif est ici de mettre en œuvre le service le plus efficace possible afin d'assurer un ravitaillement performant. Pour ce faire, de véritables techniques innovantes, propres au monde du commerce, sont investies. Ainsi, fin 1916, dans le sous-secteur de Choisy-au-Bac (Oise), une véritable étude de marché est menée pour la société parisienne des "Magasins de gros des coopératives de France " qui vient de passer un contrat avec la lère Armée $^{72}$. On y dénombre les effectifs par cantonnement, en distinguant officiers et hommes de la troupe, et en évaluant les endroits où les camions-bazars pourraient s'arrêter. Des observations viennent compléter ce compte rendu, donnant des indications plus précises sur les effectifs et leur emplacement ${ }^{73}$. Au total, plus de 4.000 soldats pourront théoriquement bénéficier de ce service. Sous l'impulsion de l'état-major, ce type d'investigation est étendu et plusieurs généraux répondent à l'enquête, parfois avec plus ou moins de précision. En cherchant à faciliter la mise en place du service et à mesurer ses potentialités, les autorités militaires en assurent également sa performance, critère essentiel dans une entreprise capitaliste moderne. D'ailleurs, dans le même esprit, afin de favoriser l'esprit d'implication, la maison « J. Damoy " alloue une prime d'intéressement aux soldats affectés à la vente des produits dans les camions-bazars ${ }^{74}$. De son côté, pour éviter toute perturbation et " conflits commerciaux » qui gêneraient le bon fonctionnement du service, l'Intendance cherche à limiter au maximum les concurrences dans les zones allouées aux camions-bazars, notamment entre grandes maisons parisiennes ${ }^{75}$.

De fait, tant du côté des autorités militaires que des maisons de commerce, la préoccupation constante est celle de l'efficacité afin, pour les premiers, de répondre à un besoin des troupes et, pour les seconds, d'assumer un service lui assurant une rentabilité

\footnotetext{
68 Ibid., Note du DES aux généraux commandants des armées, 13 avril 1915.

${ }^{69}$ Ibid., Lettre de la maison « Damoy » à l'adjoint à l'Intendance, 24 juillet 1915.

70 Ibid., Lettre de l'Intendance militaire au DES, 26 octobre 1915.

${ }^{71}$ Ibid., Lettre du DES au Commissaire régulateur de la gare de Noisy-le-Sec, 10 août 1915.

72 SHD, 19 N 170, Armées - Front occidental, lère Armée, 4e Bureau, Intendance, Dossier "Camions-bazars ", Convention passée entre le DES et le Magasin de gros des coopératives de France, 17 nov. 1916.

${ }^{73} \mathrm{Ibid}$., Compte rendu pour servir à l'établissement des itinéraires des camions-autos-bazars, 17 décembre 1916.

74 SHD, 19 N 964, arch. cit., Dossier "Personnel - Auto-bazars ", Lettre de " J. Damoy » à I'Intendance, 11 décembre 1915.

${ }^{75}$ SHD, 19 N 170, arch. cit., Lettre de l'Intendant de la lère Armée au général commandant, 25 mai 1917.
} 
S. Le Bras, "Business as usual ? Société de consommation et Grande guerre ", Histoire, économie et société, $n^{\circ} 3,2018$, p. 96-114

certaine. Dans tous les cas, c'est là une manifestation évidente de la pénétration jusqu'aux lignes de tranchée de la société de consommation.

\section{III. $\quad$ La société de consommation au révélateur de la guerre}

Dans un ouvrage paru en plein conflit, intitulé La Guerre, le commerce français et les consommateurs, le directeur de la revue Alimentation, Commerce et Industrie, Gilles Normand, évoque "le développement de pratiques commerciales modernisées " depuis quelques années, ajoutant qu'il y a " dans la fonction économique [des commerçants], un rôle éminemment social à remplir ${ }^{76}$. Ce sont là deux des fondements de la diffusion d'une " nouvelle culture de consommation ", initialement réservée à la population parisienne ${ }^{77}$, et dont le conflit permet en partie l'extension. Trois facteurs, en lien avec le cas des camionsbazars, permettent d'en prendre la mesure.

Tout d'abord, la prestation répond à un désir de consommer très répandu parmi la troupe. Chez Dorgelès, il se caractérise par des pratiques consommatrices récurrentes lors des périodes de repos. Ainsi, dans ce petit village du front qui sert de décor à l'un de ses chapitres, l'auteur dépeint de nombreuses scènes chez les marchands. Si ceux-ci sont souvent présentés de manière caricaturale et négative, renvoyant à l'image dépréciative qui existe alors à l'époque contre les " boutiquiers ${ }^{78}$ ", ils restent des acteurs majeurs et récurrents de la vie en arrière des lignes de combat. L'épicier Thomas qui avait de l'argent "plein ses tiroirs", connaissait une prospérité sans commune mesure en raison du stationnement des troupes dans le village ${ }^{79}$; des troupes qui, dès " la soupe mangée ", remplissaient les boutiques tous les soirs, sans véritablement savoir ce qu'elles vont y acheter, faisant la queue devant une boulangerie pourtant fermée ${ }^{80}$. Ici, la description faite par Dorgelès renvoie à deux registres différents qui construisent l'imaginaire du poilu : celui du loisir et celui du besoin.

Le premier est confirmé par L'Écho des marmites en février $1916^{81}$. Un article, consacré aux «plus grandes joies du Poilu » présente le plaisir qu'éprouvent les soldats lorsqu'ils stationnent dans les petites villes d'arrière-front à " s'arrêter devant les devantures des boutiques » et « se livrer aux joies de faire des courses en ville et d'acheter un tas de choses dont on n'a pas besoin ". Bien évidemment, comme chez Dorgelès, il s'agit ici d'une considération anecdotique de l'expérience combattante, celle-ci étant majoritairement construite autour du combat et du repos. Pourtant, elle est accréditée par d'autres témoignages. Chez Jacques Péricard, au moment de quitter Verdun, les hommes de son unité espèrent cantonner dans une localité où ils pourront acheter un kilo (un litre) de pinard pour I'un, une omelette de douze œufs pour l'autre, un cigare à trois sous pour un dernier. Les "trois passions maîtresses " du poilu passent ainsi par la consommation. C'est ce que confirme Louis Lecomte, chef de pièce au $32^{\mathrm{e}}$ Régiment $\mathrm{d}^{\prime}$ artillerie. De passage par Joinville (Haute-Marne), il se plaint de sa trop courte halte à dans " une petite ville où l'on [peut] se réassortir [et] acheter différentes petites choses [pour] s'offrir en passant un petit

\footnotetext{
${ }^{76}$ Gilles Normand, La Guerre, le commerce français et les consommateurs, Paris, Perrin et Cie, 1917, p. 8-12.

${ }_{77}$ Marie-Emmanuelle Chessel, Une histoire de la consommation, Paris, La Découverte, 2012.

${ }^{78}$ Voir par exemple à ce sujet G. Normand, La Guerre, le commerce... op. cit., chapitres I et II sur les petits commerçants.

${ }^{79}$ R. Dorgelès, Les Croix de bois, op. cit., p. 108.

${ }^{80}$ Ibid., p. 110-11.

${ }^{81}$ L'Écho des marmites, $n^{\circ} 8,29$ février 1916, p. 9.
} 
S. Le Bras, "Business as usual ? Société de consommation et Grande guerre ", Histoire, économie et société, $n^{\circ} 3,2018$, p. 96-114

complément de vie. " II regrette devoir quitter si rapidement la ville " très vivante, avec un assez bon commerce " qui " pouvait procurer ce petit bonheur à tous ${ }^{82}$.

Le second registre, celui du besoin, est proche du premier et parfois le recoupe, mais se singularise par une motivation différente, celui de répondre à un manque. II explique, on l'a vu, la multiplication des mercantis au front. Mais comme s'en indigne le journal syndicaliste La Bataille, c'est l'inaction des autorités face au pullulement des profiteurs de guerre au front qui en est la cause. Selon l'auteur de l'article en mars 1916, ce qui " retient certains chefs de corps [...] de leur faire fermer boutique [...] est la peur du lendemain ". Et l'auteur de formuler la question que se posent ces gradés: "Où trouver ensuite de quoi se ravitailler ? "83. D'ailleurs, les instructions visant à instaurer les camions-bazars abondent dans ce sens. Fin 1915, une circulaire du GQG, ayant pleinement intégré par le vocabulaire qu'elle emploie les logiques consuméristes, expose un dispositif commercial "ayant pour but de favoriser l'apport dans les cantonnement d'une plus grandes quantité de marchandises, afin de mieux équilibrer l'offre et la demande ${ }^{84}$. "

Dans les deux cas, loisir et besoin, l'adéquation à cet imaginaire découle d'un impératif fondamental : assurer le bon moral des soldats. Cet enjeu, essentiel dans un contexte de guerre, est d'ailleurs relevé par les autorités elles-mêmes. En juin 1917, dans une note relative au fonctionnement des coopératives de consommation au front, il est question de " satisfaire aux besoins matériels de la troupe et de contribuer ainsi à maintenir son état moral ${ }^{85}$. " Le lien entre consommation et moral est ainsi clairement reconnu. II l'était déjà implicitement et plus spécifiquement dans le cadre des camions-bazars - dès 1915. En effet, à la fin de l'année, suite à l'expérimentation des wagons-bazars auprès du $18^{\mathrm{e}}$ corps, d'autres unités, ayant eu vent du dispositif mis en place et de son fonctionnement, réclament son extension ${ }^{86}$. En 1916, les doléances sont les mêmes : en avril, un capitaine de la $49^{\mathrm{e}}$ compagnie d'aérostiers réclame la visite par les camions-bazars d'un cantonnement où ne se trouve qu'un épicier « ne donnant pas satisfaction ${ }^{87}$ ». En mars 1917, une note du GQG souligne les plaintes émanant de troupes qui déplorent les inégalités dont elles sont victimes car certains cantonnements sont plus visités que d'autres par les camions-bazars ${ }^{88}$. En creux, on peut y lire le mécontentement des soldats de ne pouvoir s'approvisionner et le souci de l'état-major d'y remédier, mais également les manquements dont ce dernier est responsable en matière d'approvisionnement. Ce mécontentement, évoqué par les journaux de tranchés ${ }^{89}$, est luimême relayé par les milieux politiques. Si à de multiples reprises, le gouvernement est sollicité au sujet de la cherté de la vie au front et de son action contre les mercantis, certaines interpellations sont encore plus précises et spécifiques. En avril 1916, le député du Pas-deCalais, Narcisse Boulanger, interroge ainsi le ministre de la Guerre au sujet de l'envoi de camions-bazars dans les villages occupés par les troupes, mais désertés par les populations et

\footnotetext{
${ }^{82}$ Carnets de guerre de Louis Lecomte, Fonds privés familiaux, 17 janvier 1917.

83 "Les mercantis. L'inadmissible excuse », La Bataille, 09 mars 1916.

${ }^{84}$ SHD, 18 N 32, arch. cit., Dossier "Taxation des denrées... ", Circulaire du GQG aux généraux d'armées, aux DES et aux préfets des zones des armées, 12 novembre 1915.

${ }^{85}$ Ibid., Note du QG de la VI ${ }^{\mathrm{e}}$ Armée, 3 juin 1917.

${ }^{86}$ SHD, 19 N 964, arch. cit., Dossier « Autos-Bazars » n³, Note en exécution des prescriptions du général en chef et du DES, Ve Armée, nov. 1915.

${ }^{87}$ Ibid., Dossier « Autos-Bazars » n³, Capitaine Devaux au DES de la Ve Armée, 05 avril 1916.

${ }^{88}$ SHD, 18 N 32, arch. cit., Dossier " Taxation des denrées... ", Note de la Direction de l'Arrière, 30 mars 1917.

${ }^{89}$ En 1915, dans son premier numéro, Le Canard des boyaux dans un article sur « Les petites choses qui donnent le cafard » évoque la réception d'un mandat de 50 francs alors que toute habitation (et donc tout commerce) est à plus de $20 \mathrm{~km}$.
} 
S. Le Bras, "Business as usual ? Société de consommation et Grande guerre ", Histoire, économie et société, $n^{\circ} 3,2018$, p. 96-114

donc les commerçants ${ }^{90}$. Quelques mois plus tard, c'est au tour du vendéen Victor Rochereau d'écrire au ministre au sujet de régiments qui ne sont jamais visités par les camions-bazars ${ }^{91}$. À bien des égards, on est ici très clairement dans un processus qui veille à assurer, pour partie, le bien-être des soldats par la consommation, celle-ci participant au bon moral des soldats, soit par la distraction qu'elle propose, soit en assurant la satisfaction de besoins premiers.

Se pose dès lors la question de la réelle envergure de cette influence capitaliste et consumériste au front. Bien que difficile à mesurer, elle peut être appréhendée à travers plusieurs indicateurs.

Le premier consiste dans la portée du dispositif. En effet, dans le domaine de la consommation contemporaine et plus spécifiquement dans celui de la relation entre vendeur et acheteur, la notion de satisfaction est primordiale ${ }^{92}$. Bien qu'en réalité les intérêts des uns et des autres soient différents (faire du bénéfice pour le vendeur et, dans notre cadre, assurer l'efficacité des troupes pour l'état-major), le discours est toujours articulé autour de l'intérêt du client ${ }^{93}$. C'est d'ailleurs ce qu'indiquent les archives militaires. Dès août 1915, une lettre du général divisionnaire à la tête du $18^{\mathrm{e}}$ corps emploie le terme de " client " pour qualifier les soldats qui s'approvisionnent auprès des wagons-bazars expérimentaux de son unité. Conformément aux injonctions propres à la société de consommation, il y ajoute même les valeurs cardinales de satisfaction et de rapport qualité-prix des produits : "L'expérience des wagons-bazars faite au $18^{\mathrm{e}} \mathrm{CA}$ n'a donné lieu à aucune réclamation, ni de la part des corps, ni de celle des clients qui ont paru satisfaits de la qualité et du prix des marchandises reçues ${ }^{94}$. " Cette rhétorique est régulièrement reprise dans les correspondances ou les circulaires tout au long du conflit. En 1916, le DES de la Ve armée, dans une lettre à la maison "J. Damoy", qualifie les soldats " d'acheteurs ${ }^{95}$ ", tandis qu'en 1918, la maison parisienne en réponse à une lettre de l'Intendant, évoque "l'importance de la clientèle ${ }^{96}$ " auprès de laquelle elle fait sa publicité par le biais de ses catalogues diffusés à plusieurs milliers d'exemplaires. Très clairement, le discours adopté par l'ensemble des acteurs structurant ce dispositif est celui, classique, du commerce et, légitimement, les soldats sont donc perçus comme des consommateurs.

Par conséquent, en vertu de ce principe primordial de satisfaction, qu'en est-il de l'efficacité de ce service ? La réponse est, globalement, mitigée. Les autorités mettent très tôt en évidence la réussite des camions-bazars et leur nécessaire extension ${ }^{97}$, tandis que les différentes adaptations (comme le passage à trois vendeurs) dénotent le succès de l'entreprise. Par ailleurs, les nombreuses plaintes de soldats ou de gradés car les camionsbazars ne passent pas dans leur cantonnement ou le manque ponctuel de tel ou tel produit ${ }^{98}$,

90 JORF, Chambre des députés, question n 9650, 18 avril 1916, p. 1018.

${ }^{91}$ Ibid., question $n^{\circ} 9650,22$ sept. 1916, p. 1916.

${ }^{92}$ Voir par exemple à ce sujet André Cadet et Bernard Cathelat, "À propos de l'image du consommateur ", Les Cahiers de la publicité, vol. 16, 1966, p. 141-145 ou

${ }^{93}$ Alain Chatriot, Marie-Emmanuelle Chessel, Matthew Hilton, «Introduction » in A. Chatriot (et alii, dir.), Au nom du consommateur. Consommation et politique en Europe et aux États-Unis au XXe siècle, Paris, La découverte, 2005, p. 7.

${ }^{94}$ SHD, 19 N 964, arch. cit., « Dossier Auto Bazar » n³, Lettre du général Marjoulet, commandant le $18^{\text {e }} \mathrm{CA}$ au DES, 19 août 1915.

95 Ibid., Lettre du DES à « J. Damoy », 18 avril 1916

${ }^{96}$ Ibid., Dossier « Auto-Bazars -Affaires Instances », Lettre de « J. Damoy », 19 mars 1918.

${ }^{97}$ SHD, 18 N 32, arch. cit., Dossier « Taxation... ", Lettre du commandant en chef aux généraux d'armées et DES, 10 déc. 1915.

${ }^{98}$ Voir par exemple SHD, 19 N 964, arch. cit., " Dossier Auto Bazar » n³, Lettre du Colonel Derville au DES, 22 juillet 1916. 
S. Le Bras, "Business as usual ? Société de consommation et Grande guerre ", Histoire, économie et société, $n^{\circ} 3,2018$, p. 96-114

indiquent, en négatif, la nécessité du dispositif et un succès certain. Celui-ci est d'ailleurs confirmé dans la Ve Armée par l'extension de l'activité de la maison « J. Damoy ». À l'été 1917, la maison parisienne demande à disposer d'un deuxième entrepôt, à Germaine (Marne), ce qui lui est accordé à l'automne par les autorités ${ }^{99}$. L'existence même du service en 1917 et en $1918^{100}$ atteste de sa pérennité, alors qu'en 1916, les coopératives divisionnaires sont instaurées afin de les remplacer. Certains reportages photographiques au front, censés être le reflet de l'efficience organisationnelle de l'état-major, démontrent tout autant de la popularité de la mesure. On y voit de nombreux soldats amassés autour des camionsbazars ${ }^{101}$, certains par curiosité, d'autres pour s'approvisionner en menus objets ou, très souvent, en vin ${ }^{102}$. Par ailleurs, le nombre de catalogues distribués par la maison " J. Damoy " permet de mesurer son rayonnement : environ un catalogue est distribué par section ${ }^{103}$, ce qui n'est pas négligeable pour un service qui, dans la $V^{e}$ armée, est géré collectivement par l'officier d'approvisionnement. Toutefois, le service n'est pas sans amener critiques, ni interrogations. Elles sont bien évidemment tout d'abord formulées par les soldats euxmêmes. En 1917, dans la lère Armée, un rapport pointe les nombreux dysfonctionnements, tant en raison du manque de produits que des prestations (prix non affichés par exemple) ${ }^{104}$. Dans d'autres armées, on trouve le nombre de camions-bazars trop restreint, ces derniers paraissant «ne pas suffire aux besoins des hommes ${ }^{105}$ ". D'ailleurs, en l'état actuel de nos travaux, l'absence, dans les correspondances, les carnets ou la littérature, de références aux camions-bazars tend à laisser penser que le système, sans être un épiphénomène, fut relativement limité. En témoignent un général qui, à l'été 1917, avoue ne pas connaître l'existence du service dans la localité où il vient d'arriver ${ }^{106}$ ou cet article de La Bataille comparant les camions-bazars à des « camion[s]-fantôme[s] [...] plus certainement connu[s] de la population parisienne, qui en entend souvent parler, que des poilus qui ne le voient jamais ${ }^{107}$. „ Et de lister les troubles du système : marchandises en maigre quantité, prix élevés, passages éphémères ou lors des exercices, éloignement des combattants des premières lignes qui n'y ont jamais accès, vente au compte-goutte pour ne pas manquer de marchandises au prochain cantonnement, etc. Si le constat est rude (et motivé par la campagne forcenée que mène le journal pour les coopératives militaires, concurrentes des camions-bazars), il rejoint une réalité, elle, incontestable : en dépit de l'instauration des camions-bazars, les mercantis subsistent voire fleurissent toujours au front ${ }^{108}$, preuve $d^{\prime}$ un déséquilibre persistant (tant en quantité qu'en qualité) entre demande et offre assurée par l'état-major.

\footnotetext{
99 Ibid., Dossier « Auto-Bazars -Affaires Instances », Lettre de l'intendant de la Ve Armée au général commandant, 25 septembre 1917.

100 La maison « Olida » dispose par exemple toujours d'un contrat avec la III Armée en septembre 1918. Cf. SHD, 19 N 245, Armées - Front occidental, lère Armée, DES, Dossier " Magasin central - Olida », Lettre au général commandant d'armée, 15 sept. 1918.

${ }^{101}$ Voir par exemple Bibliothèque de documentation internationale contemporaine (BDIC), VAL 188/019 et 020, Clermont-en-Argonne (Meuse), 18 juill. 1915 ou VAL 416/176, Marcelcave (Somme), 23 sept. 1916.

102 Sur cette question, voir Charles Ridel, L'lvresse du soldat. L'alcool dans les tranchées (1914-1918), Paris, Vendémiaire, 2016.

${ }^{103}$ Fin 1915, 5.000 catalogues sont distribués tous les 15 jours pour la Ve Armée qui compte 300.000 hommes. Cela correspond donc à un catalogue pour 60 soldats, soit une section.

104 SHD, 19 N 170, arch. cit., Dossier « Camions-Bazars », Compte rendu du Lieutenant Moreau, 27 août 1917.

105 SHD, 16 N 2631, GQG, Direction de l'Arrière, Intendance, Dossier « Rapports et CR de missions », Remarques faites au cours de la tournée aux $\mathrm{VI}^{\mathrm{e}}$ et $\mathrm{X}^{\mathrm{e}}$ armées, sept. 1917.

${ }^{106}$ SHD, 19 N 964, arch. cit., Dossier « Auto-Bazars -Affaires Instances », Lettre de « J. Damoy », 7 juill. 1917.

107 « Le camion-bazar et les poilus », La Bataille, 6 avril 1916.

108 SHD, 19 N 245, arch. cit., Note de service de la lère Armée, 10 mai 1918.
} 
S. Le Bras, "Business as usual ? Société de consommation et Grande guerre ", Histoire, économie et société, $n^{\circ} 3,2018$, p. 96-114

Ce constat est d'autant plus dérangeant que, lors d'une discussion à la chambre des députés, on pointe les nombreux avantages dont disposent les maisons de commerce qui ont accepté de prendre en charge le service des camions-bazars: "facilités de transport accordées sur les chemins de fer, fourniture gratuite d'automobiles par l'armée, remise d'entrepôts sans frais » liste le député breton Louis Deschamps, reprochant les privilèges dont elles profitent, leur permettant de réaliser, " aux frais de l'État ", de substantiels bénéfices ${ }^{109}$. Ici encore, le propos se veut dur car Deschamps défend, avec véhémence, l'instauration et la généralisation des coopératives, organes directement gérés par l'armée et permettant de vendre, selon lui, à des tarifs inférieurs de $20 \%$ par rapport à ceux pratiqués par les camionsbazars. Mais il n'est pas pour autant infondé. Les sociétés qui gèrent les camions-bazars semblent réaliser en effet de larges profits. S'il n'existe pas, à notre connaissance, de données précises sur les chiffres d'affaires des camions-bazars, plusieurs indices permettent d'avancer quelques pistes. Tout d'abord, quelques informations éparses nous renseignent : en mai 1917, Le Journal indique qu'une société de camions-bazars réalise dans la zone d'une armée (sans citer ni l'une, ni l'autre) environ $300.000 \mathrm{~F}$. de chiffre d'affaires par semaine ${ }^{110}$. Ensuite, le marché se caractérise par une très forte concentration des acteurs qui s'apparente à une forme d'accaparement validé par les autorités militaires : en décembre 1915, on apprend que le GQG valide l'attribution d'armées aux grandes maisons de commerce " F. Potin " (I Armées), "J. Damoy " (IVe, Ve, VIe Armées) ou "Olida " (IIe Armée). Ces grandes maisons, assimilées à des "capitalistes organisateurs des camions-bazars ${ }^{111}$ " disposent donc de marchés réservés et fermés, particulièrement rentables, où la concurrence est absente et où seuls les mouvements de troupes viennent reconfigurer les dynamiques de marché. Par ailleurs, la longévité des partenariats, tout comme les conséquences après-guerre, attestent de l'attrait de cette activité : la société «J. Damoy" est ainsi frappée par l'impôt extraordinaire sur les bénéfices de guerre ${ }^{112}$ et les comptes d'exercices de l'année 1917-1918 indiquent une diminution du compte client de plus de $60 \%$, en raison notamment de «la fermeture de deux magasins d'armée ${ }^{113}$ » dont on mesure ici, en négatif, l'importance pendant le conflit.

Surtout, l'armée décide elle-même de rentrer dans la logique de marché en proposant son propre système, indépendant et autonome : les coopératives. Si la démarche réside en partie sur le souci de mieux ravitailler les soldats, elle est également justifiée à de très nombreuses reprises, au Parlement comme chez les autorités militaires, par les bénéfices considérables qu'elles pourront permettre de réaliser en obtenant "le maximum de rendement ${ }^{114} »$. Ces bénéfices, ensuite, profiteront aux soldats car, réinvestis dans le fonctionnement de la coopérative, ils permettront d'encore plus diminuer les prix des produits proposés. En 1916, l'état-major décide ainsi l'extension à l'ensemble de la zone des armées

\footnotetext{
109 JORF, Chambre des députés, 3 mars 1917, p. 554-556

110 Seules quelques informations éparses existent: Le Journal en mai 1917 indique que dans une armée en particulier (mais sans la citer), les camions bazars réalisent environ $300.000 \mathrm{~F}$ de chiffre d'affaires par semaine (soit deux fois plus que les coopératives). Cf. "Les mercantis sont vaincus », Le Journal, 8 mai 1917.

111 C'est l'expression utilisée dans une note en 1917. Cf. SHD, 5 N 277, Cabinet du ministre, France-Vie économique, Consommation, Note sur les coopératives militaires, 30 janv. 1917.

112 Cf. Centre des archives économiques et financières, B-0015581/1, Fiscalité-Impositions liées à la Première Guerre mondiale, Bénéfices de guerre, Établissements Julien DAMOY, Paris.

${ }^{113}$ Direction des études économiques et financières du Crédit Lyonnais, 23928/2, Ets. J. Damoy, "Rapport de MM. les commissaires aux comptes sur l'exercice 1917-1918 », p. 6. Je remercie Olivier Londeix pour le signalement de ces archives.

${ }^{114}$ SHD, 18 N 32, arch. cit., Dossier « Taxation... ", Circulaire du GQG, 2 nov. 1916. Les Armées françaises dans la Grande Guerre présentent également le succès des coopératives en insistant sur leurs chiffres d'affaires (p. 440)
} 
S. Le Bras, "Business as usual ? Société de consommation et Grande guerre ", Histoire, économie et société, $n^{\circ} 3,2018$, p. 96-114

de pratiques jusqu'ici informelles, motivé par les chiffres d'affaires que les coopératives créées dans telle ou telle unité génèrent : 4.000 .000 de $F$ annuel pour le $1^{\text {er }}$ Corps d'armée ; $3.000 \mathrm{~F}$ quotidien pour le $32^{\mathrm{e}}$; jusqu'à $12.000 \mathrm{~F}$ quotidien pour le $33^{\mathrm{e} 115}$. En dépit de certaines oppositions, notamment au niveau politique ${ }^{116}$, le principe des coopératives est donc généralisé en novembre 1916. Chaque division d'infanterie doit désormais en posséder une, selon le même mode de fonctionnement : autour d'une coopérative principale, administrée par le service de l'Intendance, des succursales dans les cantonnements approvisionnent les soldats, principalement en denrées alimentaires (très majoritairement du vin) et produits de première nécessité (achetés principalement auprès des stations-magasins ou des centres d'approvisionnement de denrées ordinaires) ${ }^{117}$.

Incontestablement, tant dans la pratique organisationnelle que dans son discours ou ses conceptions de l'approvisionnement, l'armée a bien intégré les principes de la société de consommation, avec ses avantages et ses dérives.

La guerre, par ses multiples impacts, a entraîné des conséquences profondes sur l'ensemble de la société et de l'économie françaises. De par sa durée, tant à l'arrière qu'au front, elle se caractérise par une cherté de la vie dont s'alarment, dès le tournant de 1915, simples citoyens, politiques et état-major. Pour parer à cette situation dépressionniste (tant du moral que du pouvoir d'achat), les autorités civiles et militaires prennent de nombreuses mesures visant à encadrer les prix, contenir les pratiques spéculatives et inflationnistes et assurer un meilleur approvisionnement des soldats.

L'expérience des camions-bazars s'articule autour de cette triple exigence, laquelle s'inscrit dans le nécessaire soutien au moral des soldats, socle fondamental de la victoire. Par sa durée, ses modalités et son essence même, elle participe à l'intrusion, au front, de la société de consommation. Pensé comme un outil moderne et efficace de commercialisation, le camion-bazar transporte sur le front des produits, mais également des pratiques et des représentations, qui se distillent dans l'armée française. Les soldats sont alors immergés, en pleins combats, dans l'illusion de l'abondance propre à la société de consommation, matérialisée par les prix-courants et la possibilité théorique d'obtenir n'importe quel objet (ou presque) à quelques kilomètres des combats. En témoigne un reportage photo à Guewenheim (Haut-Rhin) en 1917 : on y voit des dizaines de soldats, amassés devant les étals d'un camionbazar, contemplant les centaines d'objets en vente ${ }^{118}$ (doc. 2 ).

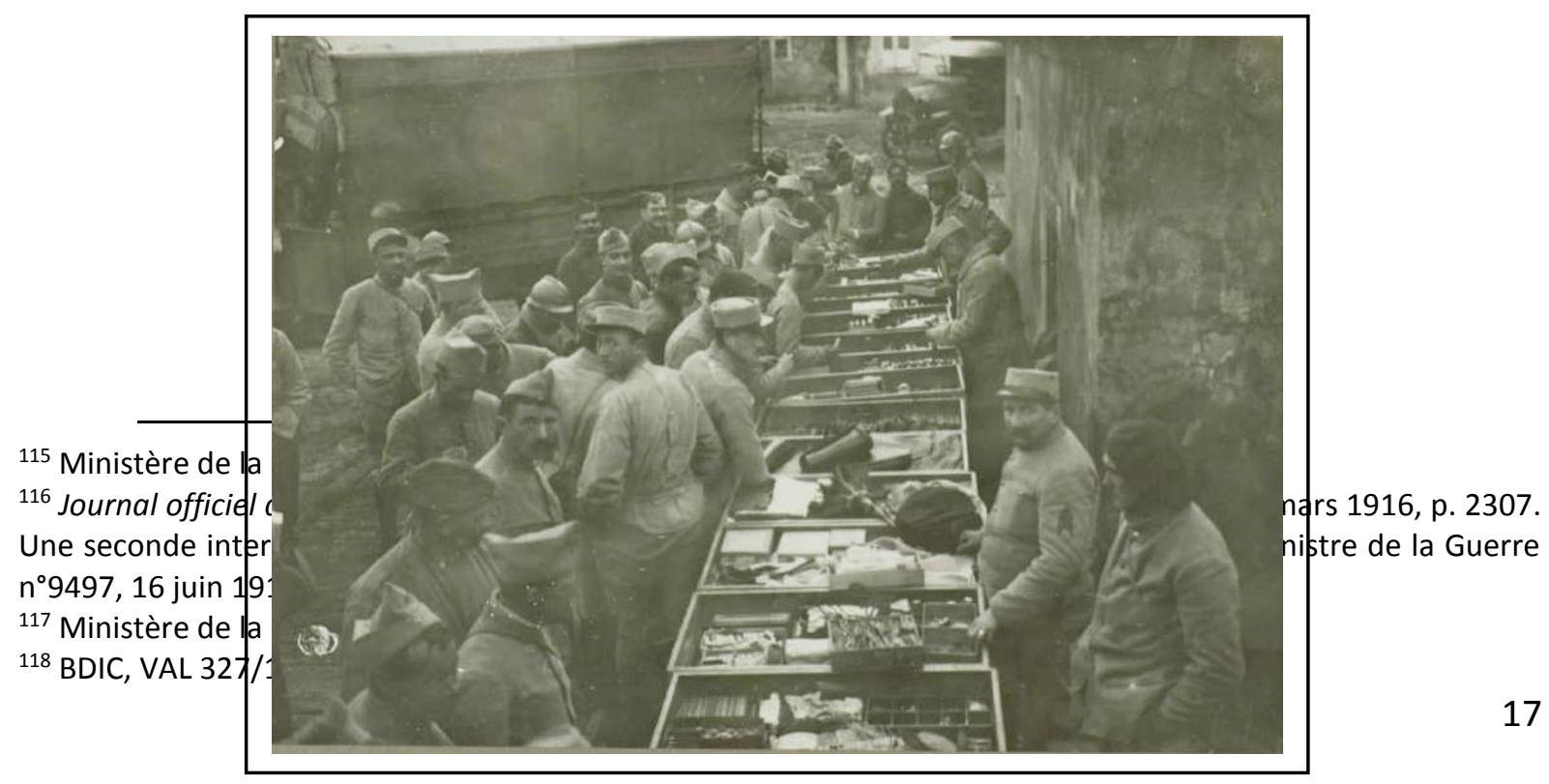


S. Le Bras, "Business as usual ? Société de consommation et Grande guerre ", Histoire, économie et société, $n^{\circ} 3,2018$, p. 96-114

Doc 2 : Un camion-bazar et ses produits dans le Haut-Rhin en 1917

Peut-être ne vont-ils rien acheter. Mais leur seule présence atteste de cette immixtion de la société de consommation au front et de la découverte de nouvelles logiques, loin de la frugalité, de la modération ou de la justesse des dépenses qui caractérisent encore alors une grande majorité de Français - et donc de mobilisés - issus de la ruralité dans les années 1910. Ainsi, plus que la réalité des pratiques individuelles (dont on ne sait finalement pas grandchose), c'est sur les représentations des soldats que ces dispositifs vont jouer, au même titre que l'imposante quantité d'un matériel militaire (casques, obus, tenues, etc.) à laquelle les poilus sont confrontés quotidiennement. La profusion devient alors un élément des processus de consommation, avec ses corollaires négatifs, gaspillage et frustration.

Certes, l'expérience connaît des limites. Surtout, au tournant de 1918, avec la reprise de la guerre de mouvement, le service est rattrapé par la réalité du conflit : en mai 1918, le magasin d'approvisionnement de Bouleuse (Marne), tenu par « J. Damoy » pour ravitailler par camions-bazars la $V^{e}$ armée, doit être abandonné sous le feu ennemi, tandis que le mouvement continu des armées rend plus complexe les relations avec les autorités et l'assurance d'une prestation de qualité119. Mais l'extension du service dans certaines armées, puis son remplacement par les coopératives militaires, généralisées pour leur part à l'ensemble des divisions d'infanterie, démontrent un souci essentiel : répondre au besoin de consommation des soldats.

Par ailleurs, une fois la guerre terminée, le service des camions-bazars de " J. Damoy " est abandonné, mais son responsable au sein de la société, M. Prouillac, fort de son expérience, est chargé « du service du ravitaillement des régions libérées ${ }^{120}$ ". Dès la sortie de la guerre, de nouvelles succursales y sont implantées, à Rilly, Fismes, Damery, Ville-enTardenois par exemple, c'est-à-dire dans la zone où avait cours le service des camions-bazars de la société ${ }^{121}$. Un service commercial en remplace donc, naturellement, un autre.

À ces différents égards, la guerre - à travers ici l'exemple des camions-bazars s'apparente bel et bien à un formidable accélérateur de l'histoire, dans une période clé de transformation des pratiques collectives consuméristes et de diffusion d'un idéal social en partie fondé sur celles-ci ${ }^{122}$, préfigurant assurément les mutations à venir en matière de

\footnotetext{
119 SHD, 19 N 964, arch. cit., Dossier «Personnel - Auto-bazars ", Correspondance entre « J. Damoy " et I'Intendant, juin-août 1918.

${ }^{120}$ Direction des études économiques et financières du Crédit Lyonnais, Ets. J. Damoy, « Rapport du conseil d'administration ", 23 déc. 1918, p. 6.

121 Ibid., Rapport du conseil d'administration, 28 déc. 1920, p. 3.

${ }^{122}$ Voir à ce sujet, Frank Trentmann, Empire of Things. How We Became a World of Consumers, from the Fifteenth Century to the Twenty-First, Londres, Penguin Books, 2016 ou Peter N. Stearns, Consumerism in World History The Global Transformation of Desire, New York, Routledge, 2006.
} 
S. Le Bras, "Business as usual ? Société de consommation et Grande guerre », Histoire, économie et société, $n^{\circ} 3,2018$, p. 96-114

consommation, notamment " de masse » (standardisation, réponse à un besoin, importance du service) $)^{123}$.

${ }^{123}$ Sophie Chauveau (dir.), "Consommer en masse ", Vingtième Siècle. Revue d'histoire, n 91, 2006. 